\title{
Jules Verne : un processus d'écriture sous contraintes
}

\section{Philippe Scheinhardt}

\section{(2) OpenEdition}

12 Journals

\section{Édition électronique}

URL : http://journals.openedition.org/genesis/636

DOI : 10.4000/genesis. 636

ISSN : 2268-1590

\section{Éditeur :}

Presses universitaires de Paris Sorbonne (PUPS), Société internationale de génétique artistique littéraire et scientifique (SIGALES)

\section{Édition imprimée}

Date de publication : 30 octobre 2011

Pagination : 173-186

ISBN : 978-2-84050-804-5

ISSN : 1167-5101

\section{Référence électronique}

Philippe Scheinhardt, «Jules Verne : un processus d'écriture sous contraintes », Genesis [En ligne], 33 | 2011, mis en ligne le 23 octobre 2013, consulté le 30 avril 2019. URL : http:// journals.openedition.org/genesis/636 ; DOI : 10.4000/genesis.636 


\title{
Jules Verne : un processus d'écriture sous contraintes
}

\author{
Philippe Scheinhardt
}

$\mathrm{L}$ 'Avertissement de 1867, au seuil du volume in-80 des Voyages et aventures du capitaine Hatteras, est l'acte de fondation du cycle romanesque des Voyages extraordinaires, concentré en son ambitieuse conclusion à la manière d'un programme : « $[\ldots]$ résumer toutes les connaissances géographiques, géologiques, physiques, astronomiques, amassées par la science moderne, et refaire sous la forme attrayante et pittoresque qui lui est propre l'histoire de l'univers'. » Quadrillage du globe et inventaire des sciences, en somme. Le dispositif contraignant du «plan »statue sur les fins du programme des Voyages extraordinaires, à la fois le littéraire et le scientifique, le didactique et l'encyclopédique. On se tromperait toutefois en pensant que ce dispositif puisse constituer un champ de réflexion suffisant sur les moyens mobilisables à cet effet par la création littéraire. Le constat s'impose plutôt d'un romancier pour qui la perspective d'un tour du monde en quatre-vingts volumes ne peut se concevoir encore que sous le signe de l'indétermination de l'œuvre à faire. «Ce que je voudrais devenir avant tout, c'est un écrivain, louable ambition que vous approuverez pleinement ${ }^{2} »$, confie-t-il à Pierre-Jules Hetzel. Bien comprendre le sens intrinsèque de cette confidence épistolaire, c'est reconnaître que cette «louable ambition » est fondatrice du rapport que Jules Verne entretient avec l'écriture littéraire avant même toute considération sur les conditions de possibilité de la somme des Voyages extraordinaires.

Dès lors que la création romanesque médiatise d'abord l'exigence du « devenir écrivain », Jules Verne ne pouvait que rencontrer le problème de «l'indétermination de l'œuvre $^{3}$ », se posant à lui comme l'horizon infini de cet enjeu identitaire. C'est seulement au terme du trajet parcouru pendant les premières années de contrat avec les éditions Hetzel, marquées par des publications célèbres de 1863 à 1866, de Cinq Semaines en ballon aux Enfants du capitaine Grant, que se dégage une distance rétrospective suffisante pour le romancier qui en vient alors à réfléchir sur les perspectives du projet romanesque lui-même. Dans l'Avertissement de 1867, si l'appel à la caution $\mathrm{du}$ 《roman scientifique » apparaît comme une contrainte de genre au premier abord, le contrat didactique encyclopédique qu'annonce l'éditeur n'engage pas son auteur dans un programme défini à l'avance. À ce moment-là, il n'existe aucune base prévisionnelle, sous la forme lâche d'un plan de travail par exemple, qui prétendrait guider le romancier au moins de façon indicative. Rappelons la liste des romans faits et à faire établie pour mémoire par Honoré de Balzac pour la programmation des chantiers génétiques de la Comédie humaine. Rien de tel dans la fabrique des Voyages

1. Avertissement de l'éditeur, signé J. Hetzel, préface aux Voyages et aventures du capitaine Hatteras, Paris, Hetzel, 1867. La genèse de cette « prière d'insérer » est passée par plusieurs étapes rédactionnelles : l'éditeur n'a imposé son style qu'après que Jules Verne et Jean Macé lui ont proposé leur version du discours promotionnel pour le lancement des Voyages extraordinaires. J'ai étudié cette genèse par le détail dans ma thèse, par comparaison avec l'Avant-propos de la Comédie humaine. Voir Jules Verne. Génétique et poïétique (1867-1877), [s.1.], [s.n.], 2005, p. $115 \mathrm{sq}$. (thèse de doctorat, Littérature et civilisation latines, Université Paris III, 5 février 2005).

2. Lettre de Jules Verne à Pierre-Jules Hetzel, en date du 25 avril 1864, $\mathrm{BnF}$, archives Hetzel, NAF 17004, $\mathrm{f}^{\circ} 6$; Correspondance inédite de Jules Verne et de Pierre-Jules Hetzel (1863-1886), Olivier Dumas, Piero Gondolo della Riva, Volker Dehs, t. I, Genève, Slatkine, 1999, lettre 7, p. 28.

3. Voir Nathalie Heinich, Être écrivain, Paris, La Découverte, 2000, p. $61 s q$. 
extraordinaires dont la forme et le sens ne se présentent à Jules Verne qu'au cours du cheminement même de l'œuvre vers l'illusoire complétude 4 .

Il semble que Jules Verne essaie de conjurer l'incertitude provoquée par l'ouverture de l'œuvre des Voyages extraordinaires, grâce à une dialectique de cette contrainte externe avec des contraintes internes. Par une sorte de paradoxe, ces dernières interviennent dans le domaine réservé de l'écrivain, celui du processus d'écriture. La régulation par des rites de l'activité poḯtique traduit de la façon la plus sensible ce domaine des contraintes, en raison du caractère public, en tout cas communicable, des conditions d'écriture comme de lecture que se donne Jules Verne pour libérer l'acte créateur.

Nous nous intéresserons donc aux phases du processus d'écriture présentées par Jules Verne lors de certains entretiens, comme représentatives de sa méthode de travail. Les étapes de cette genèse littéraire se dégagent d'un discours auctorial qui concorde dans une certaine mesure avec les témoignages laissés par les traces manuscrites. Malheureusement, les fonds conservés par la bibliothèque de Nantes, plus récemment par celle d'Amiens, n'apportent qu'un témoignage partiel puisque n'ont été conservées en général que les mises au net corrigées avant épreuves.

À cette restriction s'ajoute que Jules Verne ne communique rien aux journalistes de ses relations avec son éditeur, dont la correspondance comme les commentaires en marge des manuscrits remettent en mémoire le problème crucial de la censure, sinon de la tutelle de Pierre-Jules Hetzel sur un auteur avec qui il a entretenu des relations intersubjectives imaginaires, parfois qualifiées de «contrat fantasmatique ». La discussion sur le dénouement du Tour du monde en quatre-vingts jours constitue à cet égard un exemple de l'évolution des rapports de force depuis les débuts du romancier dans cette maison d'édition en 1862.

\section{Les rites de création}

«J'ai eu la chance d'entrer dans le monde à un moment où il existait des dictionnaires sur tous les sujets possibles. Il me suffisait de trouver dans le dictionnaire le sujet sur lequel je cherchais un renseignement, et voilà5. »
Fréquentant les bibliothèques depuis 1852, Jules Verne adopte une attitude face à l'évolution des pratiques dans la culture de l'écrit qui ne procède pas d'une posture antiromanesque, comme dans le débat entre novel et romance. Rien de semblable à une posture réaliste qui réprime les élans de l'imagination grâce à la rigueur de l'enquête scientifique et qui recherche dans le dictionnaire et l'encyclopédie le garant d'une vérité historique du présent. Jules Verne ne surestime pas l'esthétique du « document humain » chère aux frères Goncourt ; il n'en attache pas moins une valeur au « document» consubstantiel à son investissement dans le roman scientifique, dans la mesure où son manque d'éducation scientifique impose la nécessité d'une formation autodidacte dans laquelle est impliqué l'accès à un matériau culturel ou intellectuel de référence. Documentenseignement, document-renseignement : «J'ai toujours avec moi un carnet, et comme ce personnage de Dickens, je note d'emblée tout ce qui m'intéresse ou qui pourrait me servir pour mes livres ${ }^{6}$. » Le romancier s'installe ainsi dans la posture quelque peu excentrique du reporter de Pickwick Club qui ne cesse de prendre des notes, à la recherche d'une vérité documentaire de l'expérience.

L'acte de lecture comme celui de l'écriture participent chez Jules Verne des rites de l'écrivain professionnel7. Ils s'inscrivent en un temps et un espace sociaux dont

4. En revanche, une Note des éditeurs en bas de page de l'édition d'Hector Servadac, Voyages et aventures à travers le monde solaire, publiée en 1877, ressemble beaucoup plus à cette liste des romans faits et à faire. Elle se présente comme un bilan d'étape. Pierre-Jules Hetzel annonce à son public que le programme des Voyages extraordinaires continue tranquillement sa route, de volume en volume, même si l'éditeur peine dans la justification de l'idéologie positiviste prônée en 1867 , au moment où il publie cette fantaisie très subversive quant à la relation entre science et fiction.

5. Dans Robert Sherard, «Jules Verne, sa vie et son travail racontés par lui-même » [1893] (1894), dans Entretiens avec Jules Verne 1873-1905, Genève, Slatkine, 1998, p. 92. Pour les notes de référence de ces entretiens, les crochets indiquent la date de la visite, les parenthèses la date de la publication.

6. Ibid., p. 91.

7. Sur ces aspects généraux de l'écrivain à l'œuvre, voir ma collaboration avec Daniel Compère dans le catalogue de l'exposition organisée par la ville de Nantes en 2000 : Jules Verne écrivain, Nantes, Bibliothèque municipale/Coiffard Joca Seria, 2000, p. 33-49. 
peuvent témoigner les diverses visites, comme celles des journalistes anglo-saxons Marie A. Belloc et Robert Sherard 8 à la maison amiénoise désormais célèbre du 2 , rue Charles-Dubois. L'austérité monacale de son bureau, « toute petite chambre grande comme une cellule »; la rigueur de l'emploi du temps occupé de cinq à onze heures au maniement de la plume sur le papier découpé et rangé sur le coin de la table ; l'ordre soigneux de ce « domaine privé » de l'écrivain organisé pour le seul travail de l'écriture : autant de signaux surprenants pour le visiteur encombré d'imageries romantiques sur la ressemblance du créateur avec ses créatures. Ce règlement de l'espace et du temps traduit pour l'activité de l'écrivain un positionnement poïétique. Professionnalisé par ses contrats avec son éditeur, Jules Verne ne peut envisager la création littéraire qu'à l'intersection de l'art et du métier, à la façon d'un travail qui suppose une discipline de vie pour que puissent être conciliées la contingence des exigences matérielles et la nécessité de l'exigence spirituelle d'un investissement maximum de la personne dans l'instauration de l'œuvre. Toute la vie de l'écrivain participe donc de ces rites qu'il invente à la mesure de ses valeurs esthétiques. Évoquer l'association de son « sang breton » et de son goût monacal pour le « cloître » propice au bonheur « d'une vie tranquille d'étude et de travail », c'est cautionner une imagerie analogue à celle de l'écrivain amoureux de la mer qui se retire de l'agitation parisienne pour les plaisirs de la solitude à bord du Saint-Michel ${ }^{9}$.

Cet espace ne constitue toutefois que l'aspect le plus socialisé des conditions imposées à son travail par l'écrivain qui s'emploie, dans les limites de ce temps horaire, à organiser une autre temporalité plus personnelle, celle du processus de son écriture : c'est l'objet de la «méthode de travail ». À partir des années 1890, Jules Verne n'a cessé d'exposer à ses interlocuteurs ses pratiques d'écriture qui correspondent à cette part de la technè qui peut d'autant mieux être communiquée au public que n'y est engagé rien d'essentiel sur l'alchimie même de l'art et de la fiction. Dans l'ensemble, la plupart des discours de cette période des entretiens permettent de relever des constantes. Ils fournissent des renseignements utiles quoique relatifs à une situation d'énonciation.

Il est ainsi possible de relever par quelles étapes essentielles doit passer la genèse littéraire pour Jules
Verne. Premièrement : un synopsis décide, au cours d'une phase de conception, de la trame d'une intrigue romanesque dont il faut déterminer, avant toute programmation, « le début, le milieu et la fin », à défaut de quoi il y a abandon du «projet ». Deuxièmement : sans établissement préalable d'un plan général, un plan de chapitres témoigne d'une phase de structuration caractérisée par un découpage scénarique général qui vise à préparer la genèse manuscriptique de la rédaction. Troisièmement : c'est le moment de l'écriture du brouillon, lequel, organisé autour du bloc graphique, limité par une «marge d'une demi-page pour les corrections », est destiné à être utilisé deux fois dans cette phase de textualisation, puisque après « la première version au crayon », la plume « repasse à l'encre » et efface les traces de la rédaction initiale pour inscrire le texte de la seconde version. Quatrièmement : avec le « premier jet d'épreuves », commence le «vrai travail» de réécriture minutieuse du manuscrit qui peut demander jusqu'à « huit ou neuf relectures d'épreuves », avant que puisse être menée à terme cette phase de finition ${ }^{10}$.

8. Robert Sherard effectue son interview pour le compte de la revue américaine McClure's Magazine et Marie A. Belloc pour la revue anglaise The Strand Magazine.

9. Jules Verne a possédé trois voiliers tous baptisés Saint-Michel : un sloop (1868, Le Crotoy), un cotre (1876, Le Crotoy), un steam-yacht (1877, Nantes). Ces navires ont constitué comme une résidence secondaire pour Jules Verne, à la fois au plan maritime et littéraire. Pour l'anecdote, Voyage sous les eaux a été écrit à bord du premier de ces voiliers aménagé en «cabinet de travail » à partir de l'été 1868. Voir Jules Verne en mer et contre tous, textes rassemblés et présentés par Philippe Valetoux, Paris, Magellan, coll. « Traces \& fragments », 2005.

10. Sur le témoignage des manuscrits du fonds nantais, il faut avouer qu'une validation de ce discours auctorial se heurte à la difficulté de la disponibilité restreinte de l'ensemble des traces manuscrites, en raison de la destruction ou de la disparition de ces documents, parfois du vivant même de l'écrivain. La question de la phase de finition ne peut être résolue par le seul exemplaire d'une épreuve de Autour de la Lune. Quant aux phases rédactionnelles, il faut différencier les deux périodes, avant et après 1876 environ, puisque auparavant il rédigeait les deux états sur deux supports différents, comme l'atteste le cas du Tour du monde en quatre-vingts jours. Les manuscrits du fonds amiénois autorisent en revanche une meilleure investigation sur la phase préparatoire en raison de la présence de fiches et de carnets dans la collection. Le colloque international « Jules Verne. De la création à la réception », organisé par le CERR de l'université de Picardie les 17-20 novembre 2010 à Amiens, a permis à cet égard une présentation de certains résultats de recherche en relation avec cette collection. 
C'est sur la base des fonds manuscrits de grands romanciers du $\mathrm{xIX}^{\mathrm{e}}$ siècle que la critique génétique a défini théoriquement les phases d'un processus d'écriture pour le champ du manuscrit moderne et a établi à titre opératoire des tableaux représentatifs d'une genèse complète11. Il faut constater que Jules Verne n'utilise qu'une partie très restreinte des possibilités offertes par ce modèle idéal. Pour le romancier des Voyages extraordinaires, la phase " prérédactionnelle » importe beaucoup moins que la phase "rédactionnelle », sous ses deux aspects corrélatifs, celui du brouillon, mais surtout celui des épreuves, de sorte qu'il serait préférable de parler pour cet aspect de relecture-écriture par l'imprimé, de phase postrédactionnelle. La distinction entre «phase prééditoriale » et « phase éditoriale » ne traduit guère la réalité des pratiques génétiques de Jules Verne. C'est dès l'envoi des mises au net corrigées autographes que peut intervenir la plume allographe de Pierre-Jules Hetzel pour annoter ou corriger la rédaction vernienne, au moment donc du passage à l'impression du manuscrit prédéfinitif sur les placards.

Quelques mots sur les rapports entre ces deux personnalités dans le contexte de l'essor de l'édition au XIXe. Les interventions de Pierre-Jules Hetzel auprès de ses auteurs, quoiqu'elles respectent les règles d'un imaginaire contractuel, empiètent sur le domaine réservé de l'écrivain, lequel soit en protège l'accès, comme Hugo ou Sand, soit en tolère la visite, en raison du bénéfice attendu de la lecture d'un tiers pour la dynamique du processus de réécriture. C'est le cas de Jules Verne pour qui la tutelle éditoriale n'était pas tant considérée comme une ingérence dans ses affaires littéraires que comme la coopération d'un guide pédagogique dont il attendait l'aide et le soutien pour l'atteinte de l'objectif ultime : « devenir écrivain ». Les entretiens ne font cependant aucune mention de cette tutelle.

Jules Verne s'efforce en effet de se positionner comme artiste, à travers une insistance symptomatique sur l'activité de relecture et de réécriture. Est légitimée une éthique du métier littéraire qui associe art et travail et qui accorde une valeur au temps, comme agent de métamorphose d'une écriture en un texte valant esthétiquement par « la forme et le style ». Le meilleur indice pour-autrui de l'écrivain à l'œuvre est constitué par le nombre de jeux d'épreuves qui, à n'en pas douter, renvoie à des pratiques d'écriture dont Honoré de Balzac n'a pas médiocrement contribué à diffuser le paradigme auprès de toute la génération des romanciers d'après $1850^{12}$.

Le parallèle entre les rites de création des deux romanciers n'est pas fortuit. Dans les deux cas, c'est une contrainte externe contractuelle qui a déterminé les protocoles de leur «méthode de travail » qu'ils se sont imposés comme une contrainte interne productrice réglant la fabrique de l'œuvre. Il ne faut pas moins noter quelles différences séparent les deux romanciers : en effet, Jules Verne ne procède pas tant par réfection structurelle et amplification textuelle d'un scénariocanevas originel que par dilatation et condensation d'un synopsis le rapprochant en fin de compte davantage de Flaubert que de Balzac. Si comparaison n'est pas raison, cette allusion à d'autres rites génétiques est l'occasion d'un questionnement sur Jules Verne lui-même. Quelle différence essentielle sépare la phase rédactionnelle de la phase ultérieure destinée à effacer « les traces du manuscrit »? Il semblerait qu'au moment du processus de rédaction se concentre toute l'activité de fictionnalisation du champ de l'action, du voyage et de l'aventure, si caractéristique de la poétique de son imaginaire qui procède de la fusion du représenté et de l'imaginé dans la configuration d'un récit romanesque. Le factuel et le fictionnel sont tellement imbriqués à ce stade de la genèse que toute distance herméneutique s'abolit entre l'écrivain et le réel imaginaire en train de surgir de sa plume. Au lieu du vis-à-vis entre le sujet et l'objet de l'écriture, Jules Verne est comme dans l'illusion référentielle, capable

11. Voir Pierre-Marc de Biasi, « Vers une science de la littérature : l'analyse des manuscrits et la genèse de l'œuvre » (dans Encyclopoedia Universalis : Symposium, t. I, Paris, Encyclopoedia Universalis, 1985, p. 924-937) et La Génétique des textes (Paris, Nathan-Université, coll. « $128 », 2000)$

12. Louis-Jules Hetzel le mentionne expressément dès l'ouverture d'un « memorandum » qu'il établit en 1905 à l'intention du fils Michel Verne pour expliquer la méthode de travail de son père : «Balzac, par la suite de ses démêlés de comptes avec ses différents éditeurs, a laissé la réputation d'un auteur qui rendait tout travail difficile par la quantité effroyable de corrections, véritables remaniements qu'il faisait subir à ses œuvres pour les amener à leur état définitif. [...] À ce point de vue, il [Jules Verne] fut pire que Balzac » $\left(\mathrm{BnF}\right.$, archives Hetzel, NAF 17010, $\mathrm{f}^{\circ}$ 45-47). 
d'agir et de pâtir dans les mondes connus et inconnus parcourus par ses héros, dont il partage métaphoriquement les épreuves. "Je suis en plein dans mon sujet par 80 degrés de latitude et $40^{\circ}$ centigrades au-dessous de zéro13. » En raison de cette captation de la conscience créatrice par le procès de fictionnalisation, on comprend l'intérêt capital porté à la phase postrédactionnelle. En effet, ce n'est que lorsqu'il est en face du premier placard de son roman que Jules Verne peut se déprendre de l'emprise de l'empathie du créateur avec ses créatures, grâce à la distance de l'imprimé qui objective dans la typographie d'un texte le tracé sémiotique de l'écriture tout entier pris dans l'événement subjectif du discours. «Entre les manuscrits et la dernière épreuve de ce que je fais, vous ne pouvez pas savoir la différence littéraire qui existe14.»

$\mathrm{Si}$, à présent, l'analyse génétique revient vers les préparatifs de la phase prérédactionnelle, il ne semble pas que la pratique de Jules Verne puisse se recommander avec autant de netteté de l' "écriture à programme », comme celle de Zola15. À en croire les entretiens, Jules Verne combinerait écriture à programmation scénarique et à structuration rédactionnelle, en raison même du partage qu'il peut établir entre les phases de son processus d'écriture. Le synopsis et le plan par chapitres de la phase prérédactionnelle indiquent une programmation scénarique. Le brouillon de la phase rédactionnelle tend à montrer l'écrivain à l'œuvre dans la structuration progressive de la fiction au fil des deux «versions » manuscrites au crayon et à la plume; le souci de la forme et du style n'intervient souvent que dans la phase postrédactionnelle des épreuves. Il faut noter toutefois que ces rites de création ont été exposés dans les entretiens, une fois constituée idéalement la «méthode de travail ». Si nous prenons l'exemple de la fin du plus célèbre des romans des Voyages extraordinaires nous constaterons que le modèle est loin d'être exactement respecté.

\section{Étude de cas : le dénouement du Tour du monde en quatre-vingts jours}

Avec Le Tour du monde en quatre-vingts jours, on 1'a dit ailleurs ${ }^{16}$, nous constatons en effet que les règles que Jules Verne expose un peu trop trivialement comme une " cuisine », avec son modus operandi de recettes et d'ingrédients, ne présentent pas un caractère immuable. Pour mémoire, son idée génératrice résulte de l'alchimie qui transmute une lecture en un projet d'écriture possible où se montre l'aspect à la fois actif et passif de l'acte documentaire d'accumulation des fiches : à plusieurs reprises, Jules Verne en expose le mécanisme par le biais de l'idée du «jour fantôme » :

[...] j'ai dans la tête plein de petits bouts d'informations scientifiques. C'est ainsi qu'un jour, dans un café à Paris, alors que je lisais dans Le Siècle, qu'un homme pouvait voyager autour de la terre en quatre-vingts jours, il m'est immédiatement venu à l'esprit que je pouvais profiter d'une différence de méridien et faire gagner ou perdre à mon voyageur un jour dans son voyage. Mon dénouement était tout trouvé. L'histoire a été écrite longtemps après. Je garde à l'esprit des idées pendant des années - quelquefois dix ou quinze - avant de leur donner forme ${ }^{17}$.

Cette question du dénouement impose sa présence avec force dans la réflexion sur la genèse de la clôture narrative (voir fig. 1). En vertu du modèle de base que s'est donné le romancier, la triade début-milieu-fin (« Je ne commence jamais un livre sans savoir ce que seront le début, le milieu et la fin 18 »), on comprendra sa préoccupation en général pour la tension narrative qui caractérise les contours d'une intrigue dans la mesure où

13. Lettre de Jules Verne à Pierre-Jules Hetzel, en date du [26 juin 1863], $\mathrm{BnF}$, archives Hetzel, NAF 17004, $\mathrm{f}^{\circ} 1$; Correspondance, I, op. cit., lettre 1, p. 21 [à propos de Voyages et aventures du capitaine Hatteras]. 14. Lettre de Jules Verne à Pierre-Jules Hetzel, en date du [21 décembre 1873], BnF, archives Hetzel, NAF 17004, f 223 ; Correspondance, I, op. cit., lettre 170, p. 204 [à propos de L'Île mystérieuse].

15. Voir la typologie « écriture à programme »/《 écriture à processus » proposée par Louis Hay (« La troisième dimension de la littérature », Texte, $\mathrm{n}^{\circ}$ 5/6, 1986-1987, p. 313-328) et sa discussion et reformulation par Pierre-Marc de Biasi (La Génétique des textes, op. cit., p. 32 sq.).

16. Voir mon article «Le Tour du monde en quatre-vingts jours », dans Jules Verne écrivain, op. cit., p. 99-115.

17. Robert Sherard, « Jules Verne, sa vie et son travail racontés par luimême » [1893] (1894), Entretiens avec Jules Verne, op. cit., p. 92.

18. Marie A. Belloc, « Jules Verne chez lui » [1894] (1895), Entretiens avec Jules Verne, op. cit., p. 106. 


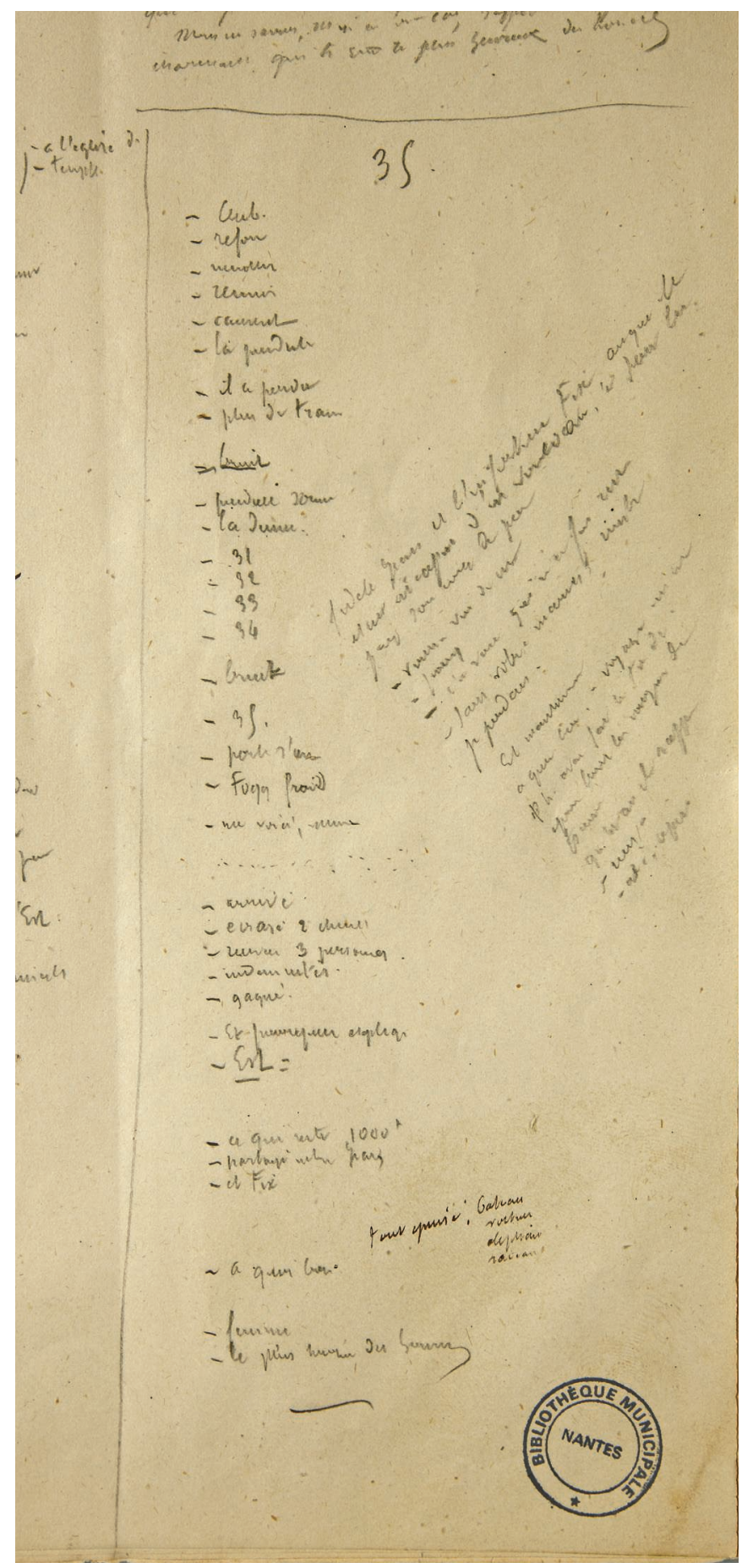

Fig. 1 : Jules Verne, Plan du chapitre XXXV. Détail du fo [48], dernier feuillet de l'ébauche-brouillon

(MJV B104, Bibliothèque municipale de Nantes) s'y attache la notion d'intérêt de lecture ${ }^{19}$. Tout dépend du mode d'approche du récit. Pour Le Tour du monde, le dénouement du «jour fantôme » coïncide avec une poétique aristotélicienne du telos qui assure à la séquentialité du nœud et des péripéties sa configuration narrative transformatrice. C'est sur cette logique de la clôture narrative du roman que divergent Jules Verne et Pierre-Jules Hetzel.

Avec le temps le guidage de l'éditeur, longtemps accepté de bonne grâce, s'avère moins formateur pour le romancier des Voyages extraordinaires. Dès lors que les contraintes de cette tutelle dérivent vers le contrôle du pouvoir créatif, la suspicion s'installe dans la dite collaboration à mesure que la liberté assumée dans le traitement du « roman scientifique » génère des résistances, commuées en blocage d'une phase génétique, lorsque s'exerce le droit de censure éditoriale ${ }^{20}$.

Pour le Tour du monde en quatre-vingts jours, Jules Verne ne saurait certes aisément accepter la discussion, tant la mise en intrigue avait conditionné la genèse scénarique du roman. C'est une flagrante méconnaissance de cette finalité du dénouement qu'affiche donc PierreJules Hetzel sur les conseils de son fils 21 dans une lettre du 8 novembre 1872 :

Jules a une assez bonne idée pour la fin du Voyage autour $d u$ monde.

Vous parlez d'une pendule indiquant les jours. Cette pendule quand il arrive ne peut ne pas être arrêtée.

19. Pour les notions narratologiques employées ici : voir Raphaël Baroni, «Tension narrative, curiosité et suspense : les deux niveaux de la séquence narrative », conférence au CRAL: « La narratologie aujourd'hui, 6 janvier 2004 »,Vox Poética, <www.vox-poetica.org/t/lna/ baronilna.html>, 2005. 20. Voir ma contribution au séminaire général de l'ITEM (1999-2000) : « Jules Verne sous la tutelle d'Hetzel. La question de la censure dans le manuscrit d'Une ville flottante », dans Genèse, censure, autocensure, Paris, CNRS Éditions, 2005, p. 59-95.

21. Pierre-Jules Hetzel (1814-1886) est le fondateur de la société « J. Hetzel et Cie » créée en 1862 et transformée en société en commandite en 1868. Son fils Louis-Jules Hetzel (1847-1930) collabore à la maison d'édition à partir de 1869. Tandis que son père dirige la partie artistique et commerciale, lui s'occupe plutôt de la partie technique jusqu'au moment de la reprise de la direction de la maison d'édition en 1886. Les deux Hetzel signent indifféremment « J. Hetzel », conformément à la raison sociale. 


\begin{abstract}
Mais les parieurs seuls doivent être en éveil - et il me semble que ce serait à eux de dénicher Fogg, d'envahir sa maison à son retour. Ils doivent faire le guet - en un mot, il faut comme contrepartie à l'émotion du départ des paris, une émotion de retour. C'est un contrepoids indispensable, logique, nécessaire. Jules a là une très bonne idée, dont il me semble qu'il faut tirer parti même pour le journal. Rêvez-en une heure et dites-moi ce qu'il faudrait que je vous renvoie du texte pour l'appliquer 22 .
\end{abstract}

Ce discours procensorial - le discours d'accompagnement par lequel sont justifiés autoritairement ou non les appels à supprimer ou à modifier tel ou tel passage intervient au moment où, corrigeant les placards en vue de la publication dans Le Temps, Jules Verne dispose d'un regard suffisamment distant sur l'économie de son discours romanesque pour qu'il évalue le caractère intempestif des conseils d'Hetzel père et fils auxquels il riposte dans la lettre du 10 :

[...] il est parfaitement vrai que quand Fogg rentre dans sa maison, il trouve sa pendule arrêtée, et il faut qu'il en soit ainsi, parce que comme cette pendule marque les jours, il verrait bien qu'il a gagné un jour, et il ne doit pas s'en douter. Quant à l'idée de Jules d'aller faire relancer Fogg chez lui, elle est destructive de tout mon dénouement. C'est au club que Fogg doit apparaître à la date fatale, c'est $a u$ club qu'on doit l'attendre. Toute l'émotion dont vous me parlez existe au retour, pendant les 3 jours qui précèdent le retour, car, ne l'oubliez pas : d'après mes changements, toute l'Angleterre se trompe avec Fix, et prend Fogg pour le vrai voleur. Ce n'est que 3 jours avant l'arrivée de Fogg, que le vrai voleur étant arrêté, toute l'attention est ramenée sur le pari, et qu'on attend Fogg avec une émotion extrême. Mais personne ne doit soupçonner qu'il est de retour à Londres. J'ajouterai même quelques mots pour bien établir que personne ne le sait, pas même ses voisins. La maison sera restée close comme par le passé. Les adversaires de Fogg, - mais vous ne vous le rappelez pas, - ont tout fait pour savoir si Fogg est en train de revenir ; ils ont lancé des dépêches en Amérique ; ils ont consulté les listes des passagers arrivés par les derniers paquebots. Je le répète, ils ont tout fait, ils enverront même observer la maison de Fogg pendant le dernier jour, mais rien, rien ne doit leur faire supposer que Fogg est revenu. C'est au club qu'ils l'attendent, et c'est au club que l'arrivée inattendue de Fogg produit tout son effet 23 .
À la différence du commentaire en marge d'un manuscrit, l'échange épistolaire explicite l'interaction verbale dans une situation de discours qui constitue les deux interlocuteurs dans une relation de symétrie argumentative absente de l'acte de lecture éditoriale. Dans ce dernier, les traces d'une réécriture n'apportent qu'une justification partielle, sans que puisse être comblé ce déséquilibre autrement que sur le mode interprétatif. Avec ces deux lettres en effet on perçoit de quelle façon Jules Verne négocie sa distance avec Pierre-Jules Hetzel quand l'enjeu du problème du dénouement commande la réfutation des arguments proposés comme solution alternative à la séquence terminale du Voyage autour du monde.

$S$ 'affrontent deux conceptions de la fin comme problème. Le discours éditorial fonde sur la suggestion de Louis-Jules Hetzel une interprétation de la rhétorique de la fiction qui ramène à la participation émotionnelle du lecteur, préstructurée par et dans le texte24 à travers le parallèle entre le début et la fin du voyage circumterrestre, constituant un «contrepoids indispensable, logique, nécessaire ». Cette interprétation est systématiquement infirmée par Jules Verne. La longueur de la réfutation signale combien l'emprise de la tutelle éditoriale demande à l'auteur la mobilisation d'une argumentation propre à le dissuader d'une idée de remaniement contraire à la configuration même de la structure narrative, pourtant décidée d'un commun accord. Attaquant sur les déficiences de la lecture hetzelienne (« ne l'oubliez pas », « vous ne vous le rappelez pas »), le romancier adopte un système de défense qui rappelle les articulations de cette séquence finale du Voyage autour du monde pour qu'il puisse être convaincu par le détail de l'action « destructive de tout [s]on dénouement » provoquée par « l'idée de Jules ». À la question de l'émotion, cette idée des parieurs en surveillance devant la maison apporte une réponse qui s'avère insatisfaisante. L'intérêt romanesque surgit de la tension que suscite le retardement stratégique

22. Bibliothèque d'Amiens, coll. Piero Gondolo della Riva ; Correspondance, I, op. cit., lettre 145, p. 180-181.

23. BnF, archives Hetzel, NAF 17004, $\mathrm{f}^{\circ}$ 175-176 ; Correspondance, I, op. cit., lettre 146, p. 181-183.

24. Sur cette notion de « rhétorique de la fiction », voir Paul Ricoeur, Temps et Récit, Paris, Éditions du Seuil, coll. « Points Essais », 1991, t. III, p. $284 s q$. 
par le suspense sur le jour perdu/gagné. Il joue sur le défaut d'information à la fois du point de vue de Fogg que trompe l'arrêt de sa «pendule » et du point de vue de Fix et de l'Angleterre que trompe l'arrestation du coupable du vol de la banque «pendant les 3 jours qui précèdent le retour ». En tant que déséquilibre affectif, l'émotion participe de cette tension intellectuelle du suspense qui l'intègre comme un facteur excitant dès lors que revient sur le pari «toute l'attention». C'est avant tout la structuration de la séquentialité narrative du dénouement qui préoccupe Jules Verne. Les changements faits ou à faire concernent les procédés qui renforcent l'incertitude entre clarté et obscurité informatives et perfectionnent la tension par laquelle l'énigme est maintenue dans le vide initial de son déchiffrement.

Évaluons les deux changements à partir des pièces génétiques ${ }^{25}$. Pour le premier processus de réécriture ( «j'ajouterai »), la comparaison entre les états génétiques reflète le débat autour de la préparation de l'énigme du retour de Phileas Fogg. Dans le premier état (une ébauche-brouillon), au sein d'un ensemble dominé par des plans de chapitres en écriture tabulaire, le même trentequatrième chapitre bénéficie d'une première esquisse de notes que double une seconde beaucoup plus développée dans laquelle surgit une opération d'écriture linéaire, un début de rédaction à l'encre correspondant au fragment phrastique disposé en colonne et divisé en trois segments dans la première notation au crayon :

\section{4}

- ainsi donc ce

- Samedi $21 \mathrm{x}^{\mathrm{b}}$

-10 h 35 du soir

- passé

- Le lendemain - les passants

- de la rue de Saville-row

- étonnés

- mais point de sorti $(e)$

- point au club.

34.

Le lendemain les habitants de Saville-row pouvaient remarquer que les fenêtres de la
- ouvertes au 1er - poin $(t)$ à l'entresol

- mais $\operatorname{poin}(t)$ quittée

- point au club

$[\ldots]^{26}$

Ce début d'incipit de chapitre rencontre un premier achèvement rédactionnel dans le second état (une mise au net corrigée), avec le paragraphe suivant :

Le lendemain les habitants de Saville-row pouvaient remarquer que les fenêtres, à l'entresol de la maison portant le numéro 7, étaient entr'ouvertes. Au rez de chaussée, tout restait clos. Quelques esprits sagaces en conclurent que Mr. Fogg avait réintégré son domicile, et ils ne se trompaient pas. Ils pouvaient voir aussi fean, quiallaitet Passepartout qui allait et venait, physionomie triste, figure désespérée 27.

C'est sur ce paragraphe que l'addition s'appliquerait, quoique cette addition s'apparente davantage à un remaniement. Elle n'intervient à l'évidence que pendant la phase de textualisation, lors des corrections sur les placards destinés à la publication commencée depuis le 6 novembre dans le « journal » Le Temps. De ce fait, il nous manque les traces génétiques, puisque la quasitotalité des placards ou épreuves a disparu des fonds manuscrits publics. Recourons donc en compensation au texte de la pré-originale en feuilleton, au début du trentecinquième chapitre :

Le lendemain, les habitants de Saville-row auraient été bien surpris, si on leur eût affirmé que Mr. Fogg avait réintégré son domicile. Portes et fenêtres, tout était clos. Aucun changement ne s'était produit à l'extérieur ${ }^{28}$.

Au lieu de l'ajout de " quelques mots », c'est à une réduction du paragraphe que procède le remaniement pour bloquer la transmission du savoir et effacer tout signe

25. Signalons une édition de William Butcher qui annote le texte et commente certaines variations à partir de la leçon des éditions et des manuscrits : Le Tour du monde en quatre-vingts jours, Paris, Gallimard, coll. « Folio classique », 2009.

26. Médiathèque de Nantes, Centre Jules Verne, MJV B104, $\mathrm{f}^{\circ}$ [48].

27. BnF, archives Hetzel, NAF 16998, f ${ }^{\circ} 160$.

28. Le Temps, 20 décembre 1872, p. 1. 
d'une transformation de l'habitat tel que la présence de l'habitant ne puisse être soupçonnée par «personne », y compris «ses voisins ».

Pour le second processus («vous vous ne le rappelez pas »), il a bel et bien déjà été effectué sur la mise au net corrigée (voir fig. 2). Au moment de l'échange des lettres du 8 et 10 novembre, Jules Verne dispose encore de ce manuscrit comme document de référence pour les corrections sur les placards du Temps. C'est donc à l'issue de la publication en feuilleton que la maison Hetzel reçoit le manuscrit, notamment Louis-Jules pour les procédures de fabrication en volume in-18, de sorte que la rigueur des délais d'édition, une fois n'est pas coutume, dessaisit Pierre-Jules de sa prérogative habituelle de commentaire ou de critique de la plume de son auteur.

36.

Dans lequel Phileas Fogg fait de nouveau prime sur le marché

Il est temps de dire ici quel revirement de l'opinion s'était produit dans le Royaume-Uni, quand on apprit l'arrestation du véritable voleur de la Banque, un certain James Strand, qui avait eu lieu 1617 décembre à Edimbourg.

Trois jours avant, Phileas Fogg était un criminel que la police poursuivait à outrance, et maintenant, c'était le plus honnête des gentlemen, qui accomplissait son excentrique voyage autour du monde.

Quel effet ! quel bruit dans les journaux! Tous les parieurs pour ou contre, qui avaient déjà oublié cette affaire, ressuscitèrent comme par magie. Toutes les transactions redevenaient valables. Tous les engagements revivaient, et il faut le dire, les paris reprirent avec une nouvelle énergie, et le nom de Phileas Fogg fit de nouveau prime sur le marché.

Les cinq collègues du gentleman, au Reform-Club, passèrent ces trois jours dans une extrême inquiétude. Ce Fogg, qu'ils avaient oublié, reparaissait à leurs yeux. Où était-il ? Le 17 décembre, jour où James Strand fut arrêté, il y avait soixante-seize jours que Phileas Fogg était parti, et pas une nouvelle de lui ! Avait-il succombé ? Avait-il renoncé à la lutte ou continuait-il sa marche, suivant l'itinéraire convenu ? Et le samedi, 21 décembre à dix heures trente cinq du soir, allait-il apparaître comme un fantôme de l'exactitude sur le seuil du salon du club?

Il faut renoncer à peindre l'anxiété dans laquelle, pendant trois jours, vécut tout ce monde de la société anglaise. On lançait des dépêches en Amérique, en asie (sic), pour avoir des nouvelles de Phileas Fogg !.. Rien ! La police elle-même ne savait plus ce qu'était devenu le détective Fix, qui s'était si malencontreusement jeté sur une fausse piste. Ce qui n'empêcha pas les paris de s'engager de nouveau sur une plus vaste échelle ! Fogg, comme les chevaux de course, arrivait au dernier tournant. On ne le pr cotait plus à cent, mais à vingt, mais à dix, et et le vieux paralytique, lord Albermale, le prenait, lui, à égalité.

Ainsi, le samedi soir, y avait-il foule dans Pall Mall, et dans les rues voisines. On eût dit un immense attroupement de courtiers établis en permanence aux abords du ReformClub. La circulation était empêchée. On discutait, on disputait, on criait les cours du Phileas Fogg comme ceux des fonds anglais. Les policemen avaient beaucoup de peine à contenir le populaire, et à mesure que s'avançait l'heure, l'émotion, croissant, prenait des proportions invraisemblables 29 .

Cette addition marginale conforte les arguments qu'oppose la lettre du 10 novembre à la procensure éditoriale, qu'ils concernent le délai de « 3 jours », le quiproquo entre le faux voleur (Phileas Fogg) et le «vrai voleur »(James Strand), le revirement de l'opinion anglaise, notamment des parieurs du Reform-Club, la recherche d'informations ("dépêches en Amérique », « liste des passagers arrivés par les derniers paquebots », observation de la maison de Saville-row «pendant le dernier jour », etc.). Et - le plus important - « l'émotion extrême » de cette attente de la perte ou du gain du pari s'articule avec une rhétorique de la tension dramatique qui entretient le suspense jusqu'au moment où l'arrivée à la «date fatale » dans le salon du Reform-Club réinstille de l'émotion mais comme facteur stupéfiant, à la mesure de la diminution de probabilité d'un succès de Phileas Fogg. Est ainsi «produit tout son effet »- de lecture, à la fois affectivement (attente du terme du délai de quatre-vingts jours) et intellectuellement (déséquilibre entre savoir énoncif et savoir énonciatif 30 sur le « jour fantôme »). Au bilan, à la question de l'émotion, Jules Verne réplique dialogiquement en contestant la réponse problématique de l'éditeur par une réponse qui à la fois résout cette question de l'émotion et la subordonne à

29. BnF, archives Hetzel, NAF 16998, $\mathrm{f}^{\circ}$ 164. La typographie en script du titre de chapitre correspond à un ajout de Pierre-Jules Hetzel. 
, thes round :

"Je vous aime," dit-il simplement. oui, en vérité, par tout ce qu'il y a de plus sacré au monde, je vous aime et suis tout

arout fut sonné. Il arriva aussitôt. Mr. Fogg tenait à vous !

$$
\text { Passepartout }
$$

encore dans sa main la main de Mrs. Aouda. comprit et sa large face rayonna- Ah ! s'écria Aouda, en portant

Mr. Fogg lui demanda s'il ne serait pas trop tard la main à son coeur, !?

pour aller prévenir le révérend Samuel Wilson de la paroisse de St Stephen.

Passepartout

fourit de son meilleur sourire.

"Jamais trop tard," dit-il

Il n'était que dix heures moins cinq.

"Ce serait pour demain, lundi ? dit-il.

- Pour demain, lundi ? demanda Mr. Fogg, en regardant bien la jeune femme.

- Pour demain, lundi !" répondit Mrs. Aouda.

Passepartout

fent sortit, tout courant.

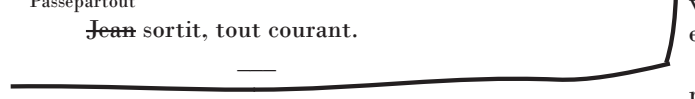

\section{6}

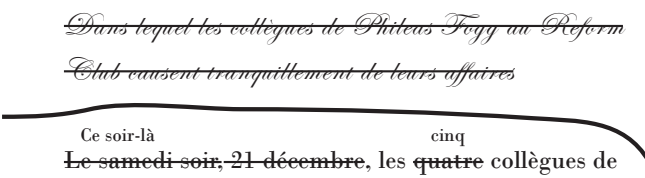

Phileas Fogg étaient réunis depuis neuf heures dans le grand salon du Reform-Club. Les deux banquiers, John Sullivan et Samuel Fallentin, l'ingénieur Andrew Stuart, Gautier Ralph, łe l'un des administrateurs de la Banque d'Angleterre, le brasseur Thomas Flanagan, tous attendaient avec anxiété.l'heure for

Au moment où l'horloge du grand salon marqua dix heures un quart, Andrew Stuart se levant, dit :

"Messieurs, dans vingt minutes, le délai convenu entre

Mr. Phileas Fogg et nous sera expiré.

- A quelle heure est arrivé le dernier train de Liverpool ? demanda Thomas Flanagan.

- A neuf heures vingt trois, répondit Gautier Ralph, et le t train suivant

n'arrive qu'à minuit dix.

- Eh bien, messieurs, reprit Andrew Stuart, si Mr. Fogg éta arrivé par le train de neuf heures vingt trois, il serait déjà ici. Nous pouvons donc considérer le pari comme gagné.

- Attendons, ne nous prononçons pas, répondit Samuel Fallentin. Vous savez que notre collègue est un excentrique de premier ordre. Son exactitude en tout est bien connue. Il n'arrive jamais ni trop tard, ni trop tôt, et il apparaîtrait ici à la dernière minute que je n'en serais pas autrement surpris.

- Et moi, dit Andrew Stuart, qui était comme toujours fort nerveux, fort impatient, je le verrais, je n'y croirais pas.

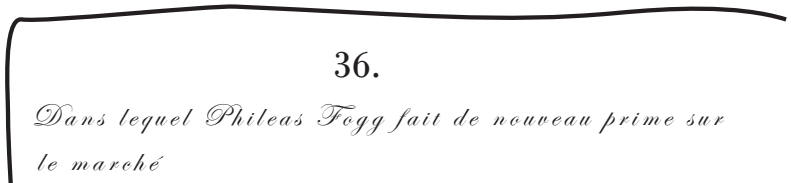

Il est temps de dire ici quel revirement de l'opinion s'était produit dans le Royaume-Uni, quand on apprit l'arrestation du véritable voleur de la Banque, un certain James Strand, qui avait eu lieu 17 décembre à Edimbourg.

Trois jours avant, Phileas Fogg était un criminel que la police poursuivait à outrance, et maintenant, c'était le plus honnête des gentlemen, qui accomplissait son excentrique voyage autour du monde.

Quel effet ! quel bruit dans les journaux ! Tous les parieurs pour ou contre, qui avaient déjà oublié cette affaire, ressuscitèrent comme par magie. Toutes les transactions redevenaient valables. Tous les engagements revivaient, et il faut le dire, les paris reprirent avec une nouvelle énergie, et le nom de Phileas Fogg fit de nouveau prime sur le marché.

Les cinq collègues du gentleman, au Reform-Club, passèrent ces trois jours dans une extrême inquiétude. Ce Fogg, qu'ils avaient oublié, reparaissait à leurs yeux. Où était-il ? Le 17 décembre, jour où James Strand fut arrêté, il y avait soixante-seize jours que Phileas Fogg était parti, et pas une nouvelle de lui ! Avait-il succombé ? Avait-il renoncé à la lutte ou continuait-il sa marche, suivant l'itinéraire convenu ? Et le samedi, 21 décembre à dix heures trente cinq du soir, allait-il apparaître comme un fantome de l'exactitude sur le seuil du salon du club ?

Il faut renoncer à peindre l'anxiété dans laquelle, pendant trois jours, vécut tout ce monde de la société anglaise. On lançait des dépêches en Amérique, en asie(sic), pour avoir des nouvelles de Phileas Fogg !.. Rien ! La police elle-même ne savait plus ce qu'était devenu le détective Fix, qui s'était si malencontreusement jeté sur une fausse piste. Ce qui n'empêcha pas les paris de s'engager de nouveau sur une plus vaste échelle! Fogg, comme les chevaux de course, arrivait au dernier tournant. On ne le cotait plus à cent, mais à vingt, mais à dix, et et le vieux paralytique, lord Albermale, le prenait, lui, à égalité.

Ainsi, le samedi soir, y avait-il foule dans Pall Mall, et dans les rues voisines. On eût dit un immense attroupement de courtiers établis en permanence aux abords du Reform-Club. La circulation était empêchée. On discutait, on disputait, on criait les cours du Phileas Fogg comme ceux des fonds anglais. Les policemen avaient beaucoup de peine à contenir le populaire, et à mesure que s'avançait l'heure, l'émotion, croissant, prenait des proportions invraisemblables. 


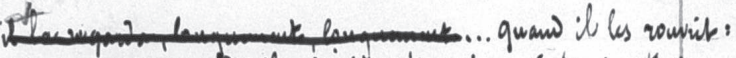

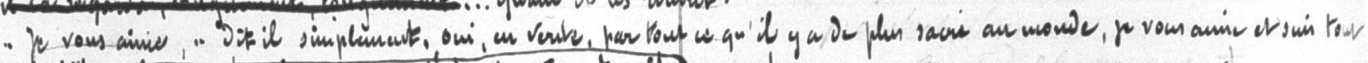

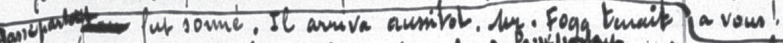

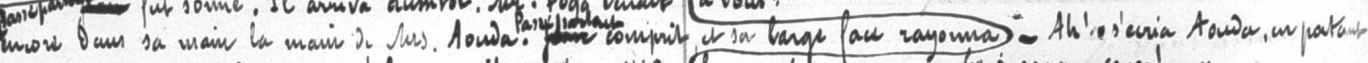

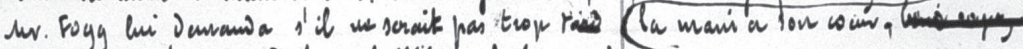

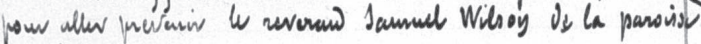

Di jo jruphow.

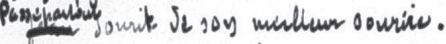

" Yamiair thop Yaw, "Jibil

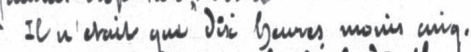

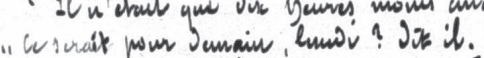

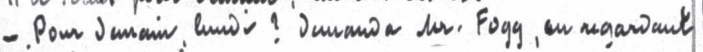

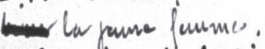

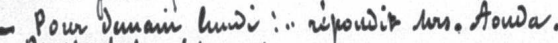

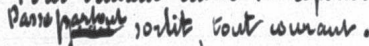

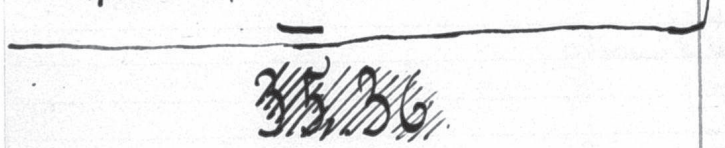

$$
\begin{aligned}
& 36 .
\end{aligned}
$$

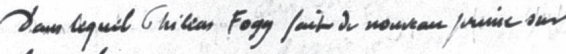

$$
\begin{aligned}
& k \text { masche. }
\end{aligned}
$$

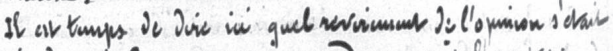

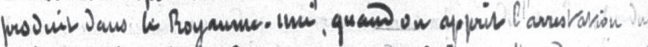

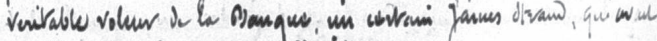

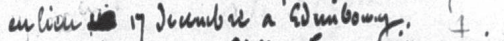

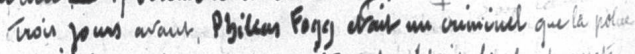

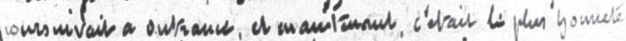

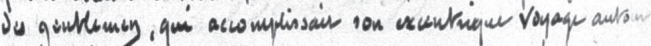
Ju monie

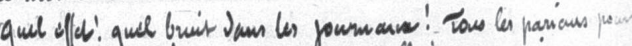

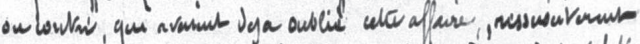

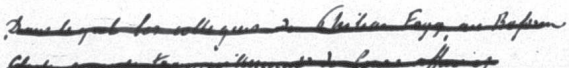

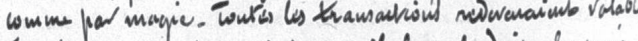

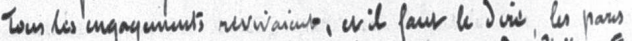

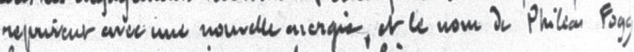
fir Ju nuw can frime sur to manifie.

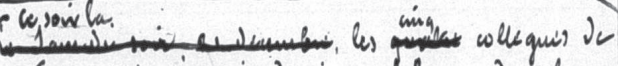

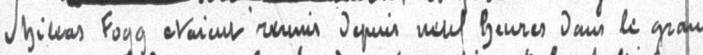

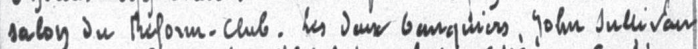

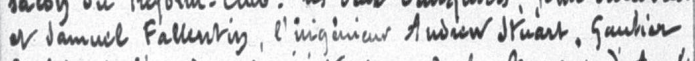

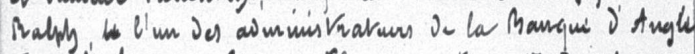

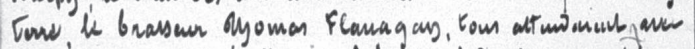

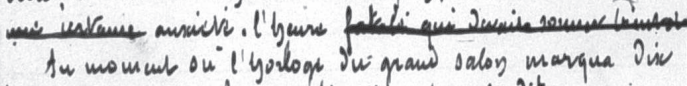

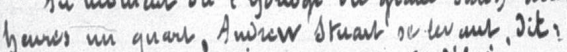

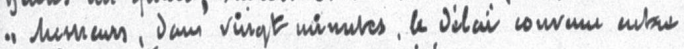
du. Phileas Foag or nous, ona eafuiri.

- A quelle Gaviri us avrive le Jumier trami do hiveppool? Dunamiar Myomas Flanayaur.

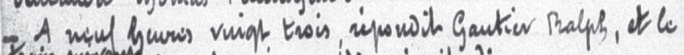

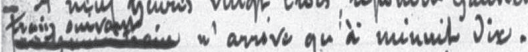

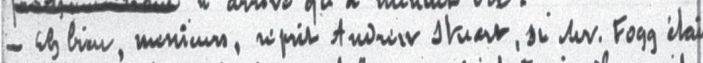
anivis par le train do neul Gaurs viniab troi it sucail

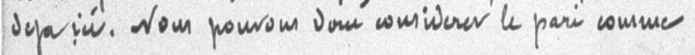
gague.

- Alruious une nous pronongom pias, ẃponsit dannul.

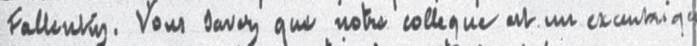

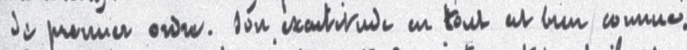
St $u$ anivis junain mi knop raw, is knop rov, it it ap.

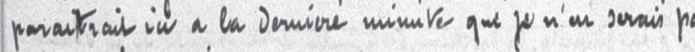
audruments juspis.

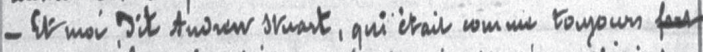

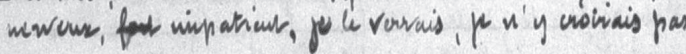

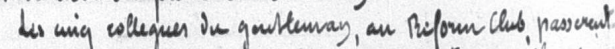

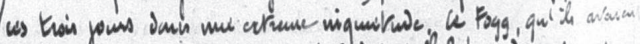

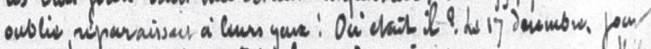

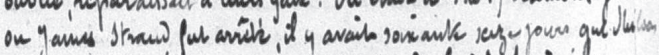

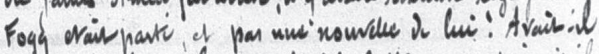

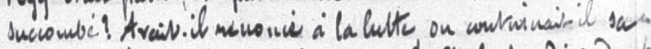

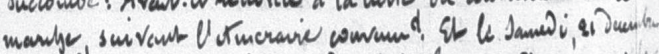

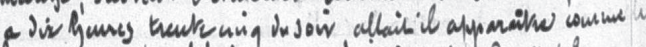

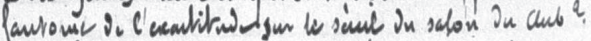

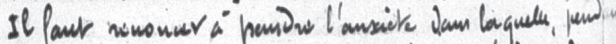

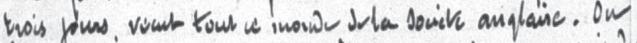

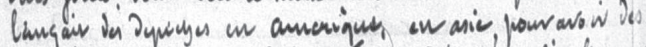

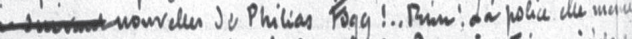

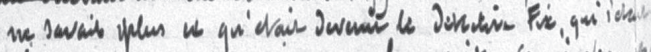

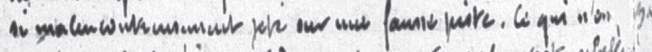

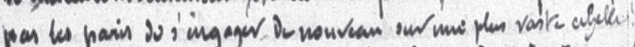

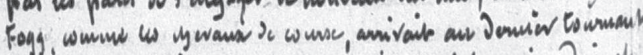

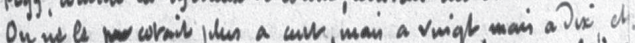

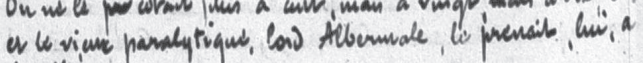

Agaliks.

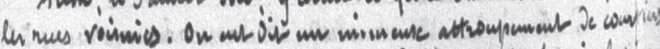

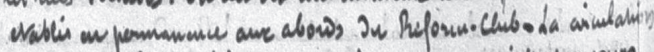

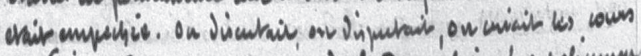

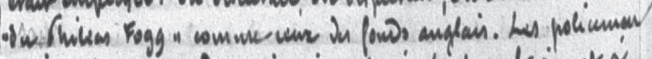

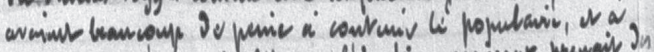

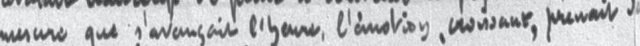
inoportion invanimblables.

Fig. 2 : Jules Verne, Le Tour du monde en quatre-vingts jours,

Mise au net corrigée (BnF, département des Manuscrits, NAF 16998, fo 164) 
la question du dénouement sur laquelle il n'y a pas à discuter. Dans ce cas particulier du Tour du monde en quatre-vingts jours, le discours de procensure, si directif soit-il (« très bonne idée »), échoue contre le barrage de l'écrivain. Il dispose du pouvoir poïétique que lui confère la maîtrise de la configuration conjointe de la fable et du sujet dans la composition d'une clôture qui ne concerne que l'intrigue romanesque elle-même. Il est vrai que Jules Verne est beaucoup plus conciliant sur les aménagements éditoriaux de la clôture textuelle ${ }^{31}$. Dénouement n'a pas la même valeur que conclusion dans la genèse des fins ${ }^{32}$ pris comme enjeu entre auteur et éditeur.

Le Tour du monde en quatre-vingts jours conserve, dans sa configuration narrative, les principes qui génèrent un projet dans l'esprit de Jules Verne, à savoir une fable et des caractères. La réflexion sur le « jour fantôme » conditionne tout le processus d'écriture du dénouement au point que l'habituelle tutelle qu'exerce l'éditeur sur la prose de «son » auteur tourne court au détriment du pouvoir procensorial de Pierre-Jules Hetzel. N'y a pas peu contribué l'histoire même de la genèse de ce récit, dans sa phase scénarique, au début de 1872, qui avait d'abord pris la forme d'une collaboration avec le dramaturge Édouard Cadol. Un contentieux n'ayant pas tardé à se déclarer entre les deux coauteurs, Jules Verne avait été amené à exposer, dans une correspondance, adressée notamment à Pierre-Jules Hetzel, les éléments essentiels de la fiction à l'appui de son argumentaire. C'est donc très vite, dégagé de la contrainte du projet de pièce grâce à la caution de son éditeur, qu'il attaque, dès le 29 mars, l'écriture du livre, le premier état de l'ébauche-brouillon, et vers le
22 juin probablement le second état de la mise au net corrigée. Pierre-Jules Hetzel, en effet, ne dispose pas du temps nécessaire pour annoter ou amender le manuscrit, dont contre toute attente on repère la présence dans le fonds Hetzel de la Bibliothèque nationale. L'absence de traces de la graphie hetzelienne dans les marges corrobore cette hypothèse : aucun envoi avant la phase de textualisation n'a été fait à Jules Verne qui a préféré l'imprimé des épreuves du Temps pour son activité de réécriture. Ce courrier du 8 novembre 1872 constitue donc le seul moment où s'exerce la tutelle de son éditeur qui, d'habitude, ne baisse pas si rapidement la garde, pour commenter, corriger, juger de l'art de «son » auteur. Faut-il y discerner la clairvoyance d'un professionnel qui a pressenti le chef-d'œuvre lors de sa lecture critique du Tour du monde en quatre-vingts jours?

30. Voir Philippe Hamon, «Un discours contraint », dans Littérature et réalité, Paris, Éditions du Seuil, coll. « Points », 1982, p. 119-181.

31. L'exemple type en est l'invraisemblance de cette reconstitution à l'identique de la robinsonnade de l'île Lincoln en plein territoire américain (Iowa), en conclusion « heureuse » de L'Île mystérieuse, après le dénouement dramatique mais si conforme à la logique de l'imaginaire vernien de dissolution des contraires dans la catastrophe finale, éruption ou explosion. Voir mon article « La mine et la cantatrice », IRIS, n ${ }^{\circ} 28,2005$, p. 219-245.

32. Voir les problèmes de terminologie discutés par Claude Duchet : « Fins, finition, finalité, infinitude », dans Genèse des fins : de Balzac à Beckett, de Michelet à Ponge, Paris, Presses universitaires de Vincennes, coll. « Manuscrits modernes », 1996, p. 5-25. 


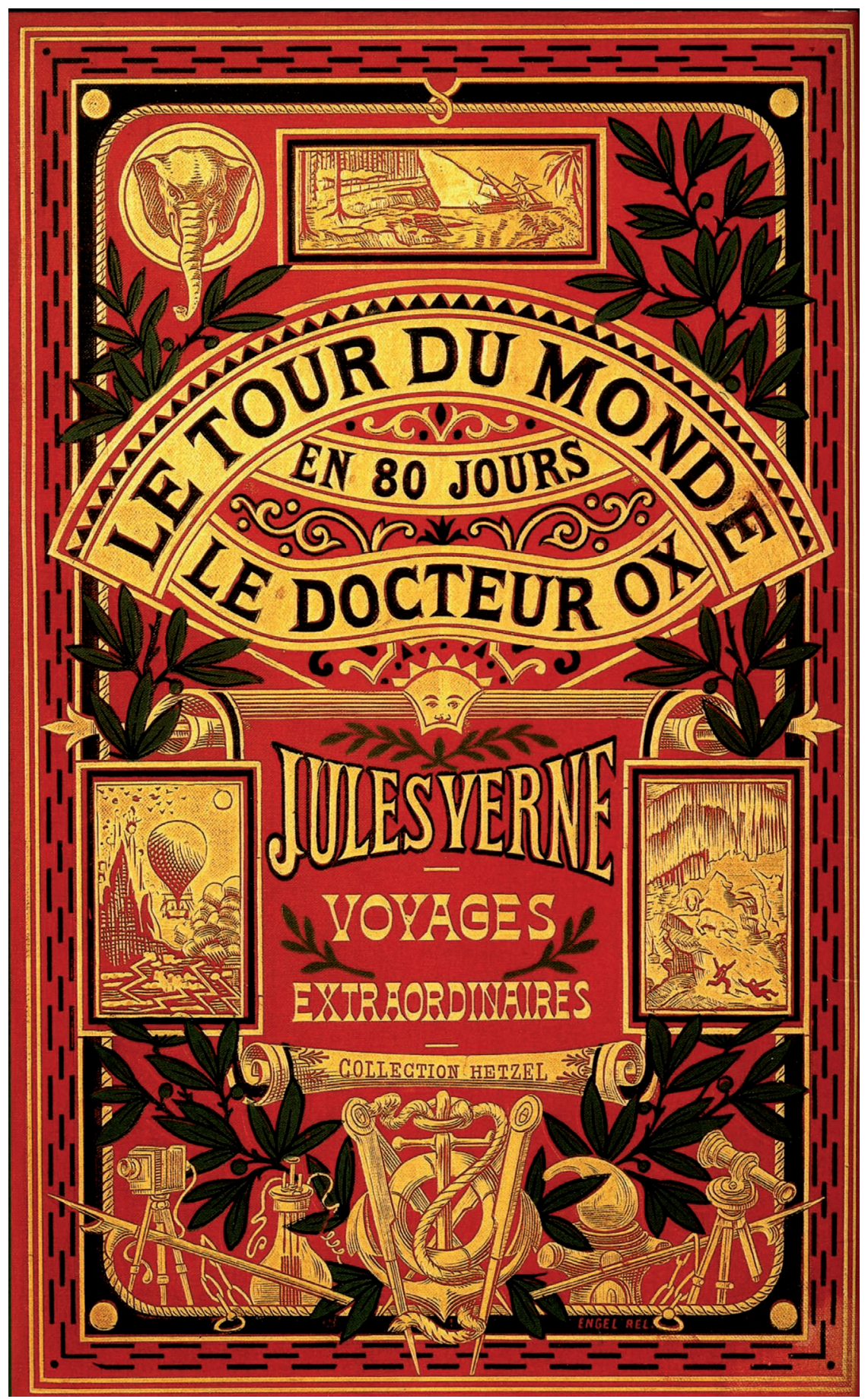

Fig. 3 : Plat du cartonnage «à un éléphant » du Tour du monde en quatre-vingts jours de Jules Verne pour l'édition in-8 illustrée sous une reliure de type polychrome « avec dos au phare » mise en service en novembre 1905 par la maison Hetzel 
Philippe Scheinhardt effectue depuis quinze ans des recherches sur Jules Verne. Sa thèse de doctorat initie une critique génétique sur l'œuvre romanesque de l'écrivain (2005). Il participe aux rencontres internationales dans un esprit de valorisation de la leçon des manuscrits trop souvent négligée par la critique. Il s'est engagé récemment dans un groupe de recherche pour une édition critique des romans des Voyages extraordinaires.

Philippe Scheinhardt, pscheinhardt@laposte.net

\section{Jules Verne : un processus d'écriture sous contraintes}

Devenu célèbre, Jules Verne reçoit souvent la visite de journalistes qui l'interrogent aussi bien sur sa vie que sur son œuvre. De ces entretiens, il ressort que l'écrivain respecte comme tant d'autres des rites de création : habitudes de documentation, méthode de genèse littéraire. Ces rites, en écho à la contrainte externe du programme encyclopédique des Voyages extraordinaires, remplissent la fonction de contraintes internes au service de l'acte créateur. Ils renseignent sur les diverses phases du processus d'écriture de Jules Verne et présentent des critères de comparaison possible avec d'autres écrivains du XIXe siècle. Il faut toutefois être prudent, en confrontant le discours avec les faits, à savoir les traces laissées par les manuscrits. C'est à ce dessein que tend l'étude génétique du dénouement dans Le Tour du monde en quatre-vingts jours où apparaît un aspect oublié par les entretiens, la tutelle exercée sur l'œuvre en chantier par son éditeur Hetzel.

After he became famous, Jules Verne was often called on by journalists who questioned him as much about his life as about his work. These interviews reveal that Jules Verne, like his fellow writers, respected the rituals of creativity: documentation habits, literary genesis methods. These rituals, echoing the external constraints of the encyclopedic program of Voyages extraordinaires, represent the constraints inherent to the creative act. They inform us on the various phases of Jules Verne's writing process and offer comparison criteria adaptable to other 19th-century writers. Caution should be exercised however, and words be confronted with facts, that is the traces left by the manuscripts. This is the purpose of the genetic study of the conclusion of Le Tour du monde en quatre-vingts jours, where an aspect overlooked in the interviews surfaces, the control imposed on the work in progress by his publisher Hetzel.

B erühmt geworden, empfängt Jules Verne oft Besuche von Journalisten, die ihn zu seinem Leben wie zu seinem Werk befragen. Aus diesen Gesprächen geht hervor, dass der Schriftsteller, wie viele andere auch, bestimme Riten des Schaffens beachtet: die Angewohnheiten des Dokumentierens, Methode der literarischen Genese. Diese Riten, Widerhall der äußeren Beschränkung des enzyklopädischen Programms der Voyages extraordinaires, erfüllen die Funktion einer inneren Begrenzung im Dienste des Schöpfungsaktes. Sie geben Auskunft über die diversen Prozessphasen von Jules Verne's Schreiben und bieten mögliche Vergleichskriterien mit anderen Schriftstellern des 19. Jahrhunderts. Man muss allerdings behutsam vorgehen bei einer Gegenüberstellung des Diskurses mit den Tatsachen, um die in den Manuskripten hinterlassenen Spuren zu erkennen. Diese Absicht verfolgt die genetische Untersuchung des Endes von Le Tour du monde en quatre-vingts jours, wo ein in den Gesprächen vergessener Aspekt ans Licht kommt, und zwar die über das im Entstehen begriffene Werk ausgeübte Vormundschaft seines Verlegers Hetzel.
Convertido en un escritor célebre, Julio Verne es visitado a menudo por periodistas que lo interrogan tanto acerca de su vida como de su obra. Esas entrevistas revelan que el escritor, como tantos otros, sigue algunos rituales de creación: hábitos de documentación, método de génesis literaria. Esos rituales, como un eco de las exigencias externas del programa enciclopédico de los Viajes extraordinarios, cumplen la función de exigencias internas al servicio del acto creador. Brindan, al mismo tiempo, información sobre las diversas fases del proceso de escritura de Julio Verne y criterios de comparación posible con otros escritores del siglo XIX. No obstante, hay que ser prudente y confrontar el discurso con los hechos, es decir, con las huellas dejadas por los manuscritos. Tal es el objetivo del estudio genético del desenlace en La vuelta al mundo en ochenta días, en el que se manifiesta un aspecto ignorado por las entrevistas: la tutela que ejerce sobre la obra en construcción el editor Hetzel.

Divenuto celebre, Jules Verne riceve spesso giornalisti che lo interrogano sulla sua vita e sulla sua opera. Questi incontri mettono in luce che lo scrittore rispetta, come molti altri, alcuni riti di creazione: abitudini di documentazione, metodo di genesi letteraria. Questi riti fanno eco alla costrizione esterna del programma enciclopedico dei Voyages extraordinaires, assolvono alla funzione di restrizioni interne al servizio dell'atto creativo. Informano sulle diverse fasi del processo di scrittura di Jules Verne e presentano criteri che possono essere comparati con quelli di altri scrittori del XIX secolo. Tuttavia bisogna essere prudenti nel confrontare questi discorsi con i fatti, cioè le tracce lasciate nei manoscritti. A questo scopo è rivolto lo studio genetico dell'epilogo di Le Tour du monde en quatre-vingts jours, dove compare un elemento dimenticato dai colloqui: la tutela esercitata sull'opera in cantiere dall'editore Hetzel.

Tornado famoso, Jules Verne recebe frequentemente a visita de jornalistas que o interrogam tanto sobre a vida quanto sobre a obra. Dessas entrevistas, ressalta que o escritor, como tanto outros, respeita os rituais da criação: hábitos de documentação, método de génese literário. Estes rituais, fazendo eco aos constrangimentos externos do programa enciclopédico das Voyages extraordinaires, cumprem a função de constrangimento interno posto ao serviço do acto criador. Por eles ficamos a conhecer as diversas fases do processo de escrita de Jules Verne e oferecem critérios de comparação possível com outros escritores do século XIX. Alguma prudência é de regra, no entanto, quando se confronta discurso com factos, nomeadamente traços deixados nos manuscritos. Nessa direcção aponta o estudo genético do desenlace de Le Tour $d u$ monde en quatre-vingts jours, em que se torna aparente um aspecto esquecido nas entrevistas: a tutela exercida pelo editor Hetzel sobre a obra em construção. 